Draft version September 21, 2021

Preprint typeset using LTEX style emulateapj v. 12/16/11

\title{
RAPID TEV FLARING IN MARKARIAN 501
}

\author{
Y. G. ZHENG ${ }^{1,2}$ AND L. ZHANG ${ }^{1}$ \\ Draft version September 21, 2021
}

\begin{abstract}
We investigate rapid $\mathrm{TeV}$ flaring in markarian 501 in the frame of a time-dependent one-zone synchrotron self-Compton (SSC) model. In this model, electrons are accelerated to extra-relativistic energy through the stochastic particle acceleration and evolve with the time, nonthermal photons are produced by both synchrotron and inverse Comtpon scattering off synchrotron photons. Moreover, nonthermal photons during a pre-flare are produced by the relativistic electrons in the steady state and those during a flare are produced by the electrons whose injection rate is changed at some time interval. We apply the model to the rapid flare of Markarian 501 on July 9, 2005 and obtain the multi-wavelength spectra during the pre-flare and during the flare. Our results show that the time-dependent properties of flares can be reproduced by varying the injection rate of electrons and a clear canonical anti-clockwise-loop can be given.
\end{abstract}

Subject headings: acceleration of particles-BL Lacertae objects: individual (Markarian 501)-radiation mechanisms:non-thermal

\section{INTRODUCTION}

Variability, which is found from radio to $\mathrm{TeV} \gamma$-ray bands, is one of the major characteristics of blazars. The variability timescales from a few minutes to days in the optical band have been extensively investigated (e.g. Sillanpää et al. 1991; Wagner \& Witzel 1996; Lainela et al. 1999). Particularly in the $\mathrm{X}$-ray and $\mathrm{TeV}$ regimes in which photons are produced by radiation of ultra-relativistic electrons close to their maximum energy, the observations of variability timescales constrain on particle acceleration mechanism in $\mathrm{TeV}$ blazars. For examples, Kataoka et al. (2001) reported the observations of the Xray flares with timescales of from hours to days for three $\mathrm{TeV}$ blazars (Markarian 421, Markarian 501, and PKS 2155-304), Albert et al. (2007) obtained a rapid $\mathrm{TeV}$ variability with of several ten minutes for Markarian 501 by MAGIC. Observed short timescales indicate that the variability is associated with small regions in the relativistic jet, which is located on a distance in excess of one hundred Schwarzschild radii $\left(r_{\mathrm{s}}\right)$ with a central black hole mass $M=10^{9} M_{\odot}$, rather than the center region (Begelman et al. 2008). Relativistic particles maybe responsible for the emission flare. These particles are ejected from the central region alone with the subsistent jet structure, and radiate away their energy at $100 r_{\mathrm{s}}$ quickly, or the particles are accelerated within the jet, close to the emission region.

Generally, soft lags can be interpreted as due to electron cooling (Kirk et al. 1998; Kirk \& Mastichiadis 1999; Kusunose et al. 2000). However, with the fast $\mathrm{TeV} \gamma$-ray flare in Markain 501 on July 9, 2005, the evidence that hard $\gamma$-ray lagged the soft ones by $4 \pm 1$ minutes was discovered (Albert et al. 2007). For explaining these abnormal phenomenon, Bednarek \& Wagner (2008) proposed the radiating blob accelerating during the flare, but in their model the particles would only undergo cooling processes without any accelerating around the high blob Lorentz factor plasma flow. Mastichiadis \& Moraitis (2008) showed that allowing the particles to accelerate gradually can explain the observed feature, and reach the particles energy to $\gamma \sim 10^{6}$, the acceleration

\footnotetext{
${ }^{1}$ Department of Physics, Yunnan University, Kunming, 650091, China (E-mail:lizhang@ynu.edu.cn)

${ }^{2}$ Department of Physics, Yunnan Normal University, Kunming, 650092, China
}

timescales is of the order of hours. Following their model, Tammi \& Duffy (2009) compared four different acceleration mechanisms, and pointed out that the timescale may be too long for first-order Fermi acceleration, so the stochastic acceleration may be as a promising candidate for the energy dependent time delays.

Motivated by above arguments, we study the timedependent one-zone synchrotron self-Compton (SSC) model in the presence of stochastic particle acceleration, and then apply the model to Markarian 501 for explaining its flare and time delay properties, especially the rapid flare of Markarian 501 on July 9, 2005. Throughout the paper, we assume the Hubble constant $H_{0}=70 \mathrm{~km} \mathrm{~s}^{-1} \mathrm{Mpc}^{-1}$, the matter energy density $\Omega_{\mathrm{M}}=0.27$, the radiation energy density $\Omega_{\mathrm{r}}=0$, and the dimensionless cosmological constant $\Omega_{\Lambda}=0.73$.

\section{THE MODEL}

Assuming the accelerated particles have an isotropic diffusion in momentum space, the evolution of the energetic particle distribution can be described by the momentum diffusion equation (Tverskoi 1967):

$$
\frac{\partial f(p, t)}{\partial t}=\frac{1}{p^{2}} \frac{\partial}{\partial p}\left[p^{2} D(p, t) \frac{\partial f(p, t)}{\partial p}\right],
$$

where $f(p, t)$ is the isotropic, homogeneous phase space density, $p$ the dimensionless particle momentum, $p=\beta \gamma, D(p, t)$ the momentum-diffusion coefficient due to interactions with magnetohydrodynamic waves, $\gamma$ the particle Lorentz factor, and $\beta$ the particle velocity in units of light velocity $c$. The particle number density $N(p, t)=4 \pi p^{2} f(p, t)$ is directly related to the phase space density.

For a specific source, after including injection, radiation, and escape of the particles, Eq. (11) can be rewritten as (Katarzynski et al. 2006)

$$
\begin{aligned}
\frac{\partial N(\gamma, t)}{\partial t} & =\frac{\partial}{\partial \gamma}\left\{[C(\gamma, t)-A(\gamma, t)] N(\gamma, t)+D(\gamma, t) \frac{\partial N(\gamma, t)}{\partial \gamma}\right\} \\
& +Q(\gamma, t)-E(\gamma, t),
\end{aligned}
$$

where we have assumed that the particles are ultra-relativistic, $\beta \approx 1$, so the momentum becomes equivalent to the Lorentz factor of particle $(p=\gamma)$. In Eq. (2), $C(\gamma, t)=(d \gamma / d t)_{\mathrm{syn}}+$ 
$(d \gamma / d t)_{\text {IC }}$ is the radiative cooling parameter that describes the synchrotron and inverse-Compton cooling of the particles at time $t$. For the synchrotron cooling, $(d \gamma / d t)_{\mathrm{syn}}=$ $(4 / 3)\left(\sigma_{\mathrm{T}} c / m_{\mathrm{e}} c^{2}\right) U_{\mathrm{B}}(t) \gamma^{2}$ is the rate of the synchrotron loss, $U_{\mathrm{B}}$ is the energy densities of the magnetic field, $m_{\mathrm{e}}$ is the electron rest mass, and $\sigma_{\mathrm{T}}$ is the Thomson cross section. For the IC cooling, the Klein-Nishina (KN) effects at high energy will be important and will modify the electron distribution and the inverse Compton spectrum (e.g., Moderski et al. 2005; Nakar et al. 2009), The rate of inverse Compton energy losses in which the KN corrections is included is given by (Moderski et al. 2005):

$$
\left(\frac{d \gamma}{d t}\right)_{\mathrm{IC}}=\frac{4 \sigma_{T} c}{3 m_{e} c^{2}} U_{\mathrm{rad}}(\gamma, t) \gamma^{2} F_{\mathrm{KN}},
$$

where, $U_{\text {rad }}(\gamma, t)=\int_{\epsilon_{0, \min }}^{\epsilon_{0, \max }} U\left(\epsilon_{0}\right) d \epsilon_{0}$ is the total energy density of the radiation field, $U\left(\epsilon_{0}\right)$ is the energy distribution of the soft photons, $\epsilon_{0}$ is the soft photons energy of the synchrotron radiation, $F_{\mathrm{KN}}=\left[1 / U_{\mathrm{rad}}(\gamma, t)\right] \int_{\epsilon_{0, \min }}^{\epsilon_{0, \max }} f_{\mathrm{KN}}(\chi) U\left(\epsilon_{0}\right) d \epsilon_{0}, \chi=$ $4 \gamma \epsilon_{0}$, the function $f_{\mathrm{KN}}$ can be approximated as (Moderski et al. 2005) as follow:

$$
f_{\mathrm{KN}} \simeq \begin{cases}1 & \chi \ll 1 \text { (Thomson limit) } \\ \frac{9}{2 \chi^{2}}\left(\ln \chi-\frac{11}{6}\right) & \chi \gg 1 \text { (KN limit) } .\end{cases}
$$

When $\chi \lesssim 10^{4}, f_{\mathrm{KN}} \simeq 1 /(1+\chi)^{3 / 2}$. Therefore, the radiative cooling parameter is given by

$$
C(\gamma, t)=\frac{4}{3} \frac{\sigma_{\mathrm{T}} c}{m_{\mathrm{e}} c^{2}}\left[U_{\mathrm{B}}(t)+U_{\mathrm{rad}}(\gamma, t) F_{\mathrm{KN}}\right] \gamma^{2} .
$$

Other terms in the left side of Eq. (2) are as follows: $A(\gamma, t)=\gamma / t_{\text {acc }}$ is the acceleration term that describes the particle energy gain per unit time, which is given by

$$
A(\gamma, t)=\frac{\gamma}{t_{\mathrm{acc}}}=\frac{2 D(\gamma, t)}{\gamma}
$$

where the acceleration time $t_{\mathrm{acc}}=\gamma^{2} / 2 D(\gamma, t)$ is used; $E(\gamma, t)$ represents escape term, which is

$$
E(\gamma, t)=\frac{N(\gamma, t)}{t_{\mathrm{esc}}}=\frac{c}{R} N(\gamma, t),
$$

where escape timescale $t_{\text {esc }}=R / c$ depends on the the emission region size $R ; Q(\gamma, t)$ is the source term, here we consider continuous injection case, i.e. the particles are continuously injected at the lower energy $(1 \leq \gamma \leq 2)$ and systematically accelerated up to the equilibrium energy $\left(\gamma_{\mathrm{e}}\right)$, where the acceleration process is fully compensated for by the cooling, i.e. $t_{\text {cool }}\left(\gamma_{\mathrm{e}}\right)=t_{\text {acc }}$.

In time-dependent one-zone SSC model, Eq. (2) needs to be solved by a numerical method because of the non-linearity process involved. We adopt an implicit difference scheme given by Chang \& Copper (1970). In our calculations, we adopt the forward differentiation in time and the centered differentiation in the energy (see Press et al. (1989) for a detail discussion). The merits of the implicit difference scheme are as follows: 1) solution is always positive; 2) particle number is always conserved; and 3) we can significantly reduces the number of mesh points in calculation with no loss of accuracy. We define the energy mesh points of electrons with logarithmic steps:

$$
\gamma_{\mathrm{j}}=\gamma_{\min }\left(\frac{\gamma_{\mathrm{inf}}}{\gamma_{\min }}\right)^{\frac{(j-1)}{j \max -1)}} ; j=1,2,3, \ldots, j_{\max },
$$

where $j_{\max }$ is the number of the mesh points, $\gamma_{\min }$ and $\gamma_{\mathrm{inf}}$ are the minimum and maximum Lorentz factors of electrons to be used in the calculation, respectively. In our calculation, a grid of 200 points has been used both for particles energy and photon frequency. Since we assume an exponential cutoff at $\gamma=\gamma_{\max }$ for the particles distribution $N(\gamma, t), \gamma_{\text {inf }}$ is taken to be much larger than $\gamma_{\max }$, here we adopt $\gamma_{\text {inf }}=10^{9}$. By defining $r=\left(\gamma_{\text {inf }} / \gamma_{\min }\right)^{\left(1 / j_{\max }\right)}$, the energy intervals can be expressed as $\Delta \gamma_{\mathrm{j}}=(r-1) \gamma_{\mathrm{j}}, \Delta \gamma_{\mathrm{j}+1 / 2}=(r-1) \gamma_{\mathrm{j}+1 / 2}=$ $(1 / 2)(r-1)\left(\gamma_{\mathrm{j}+1}+\gamma_{\mathrm{j}}\right), \Delta \gamma_{\mathrm{j}-1 / 2}=(r-1) \gamma_{\mathrm{j}-1 / 2}=(1 / 2)(r-$ 1) $\left(\gamma_{\mathrm{j}}+\gamma_{\mathrm{j}-1}\right)$ (e.g., see Park \& Petrosian 1996). Quantities with the subscript $j \pm 1 / 2$ are calculated at half grid points. In order to discretize the continuity equation, we define

$$
G(\gamma, t)=[C(\gamma, t)-A(\gamma, t)] N(\gamma, t)+D(\gamma, t) \frac{\partial N(\gamma, t)}{\partial \gamma}
$$

and $N_{\mathrm{j}}^{n}=N\left(\gamma_{\mathrm{j}}, n \Delta t\right)$. Therefore, Eq. (2) can be written as

$$
\frac{N_{\mathrm{j}}^{n+1}-N_{\mathrm{j}}^{n}}{\Delta t}=\frac{G_{\mathrm{j}+1 / 2}^{n+1}-G_{\mathrm{j}-1 / 2}^{n+1}}{\Delta \gamma_{\mathrm{j}}}+Q_{\mathrm{j}}^{n}-\frac{N_{\mathrm{j}}^{n+1}}{t_{\mathrm{esc}}},
$$

where

$$
\begin{aligned}
& G_{\mathrm{j}+1 / 2}^{n+1}=\left[C_{\mathrm{j}+1 / 2}^{n+1}-A_{\mathrm{j}+1 / 2}^{n+1}\right] N_{\mathrm{j}+1 / 2}^{n+1}+D_{\mathrm{j}+1 / 2}^{n+1} \frac{N_{\mathrm{j}+1}^{n+1}-N_{\mathrm{j}}^{n+1}}{\Delta \gamma_{\mathrm{j}+1 / 2}}, \\
& G_{\mathrm{j}-1 / 2}^{n+1}=\left[C_{\mathrm{j}-1 / 2}^{n+1}-A_{\mathrm{j}-1 / 2}^{n+1}\right] N_{\mathrm{j}-1 / 2}^{n+1}+D_{\mathrm{j}-1 / 2}^{n+1} \frac{N_{\mathrm{j}}^{n+1}-N_{\mathrm{j}-1}^{n+1}}{\Delta \gamma_{\mathrm{j}-1 / 2}} .
\end{aligned}
$$

In this case, we have $N_{\mathrm{j}+1 / 2}=(1 / 2)\left(N_{\mathrm{j}+1}+N_{\mathrm{j}}\right), A_{\mathrm{j}+1 / 2}=$ $(1 / 2)\left(A_{\mathrm{j}+1}+A_{\mathrm{j}}\right), C_{\mathrm{j}+1 / 2}=(1 / 2)\left(C_{\mathrm{j}+1}+C_{\mathrm{j}}\right), D_{\mathrm{j}+1 / 2}=$ $(1 / 2)\left(D_{\mathrm{j}+1}+D_{\mathrm{j}}\right)$, and $N_{\mathrm{j}-1 / 2}=(1 / 2)\left(N_{\mathrm{j}}+N_{\mathrm{j}-1}\right), A_{\mathrm{j}-1 / 2}=$ $(1 / 2)\left(A_{\mathrm{j}}+A_{\mathrm{j}-1}\right), C_{\mathrm{j}-1 / 2}=(1 / 2)\left(C_{\mathrm{j}}+C_{\mathrm{j}-1}\right), D_{\mathrm{j}-1 / 2}=(1 / 2)\left(D_{\mathrm{j}}+\right.$ $\left.D_{\mathrm{j}-1}\right)$. With the energy interval $\Delta \gamma$ and time interval $\Delta t$, using the no-flux boundary condition (Park \& Petrosian 1995), Eq. (2) can be written in a tri-diagonal matrix and can be solved by numerical approach (e.g. Press et al. 1989). If the electron number density $N\left(\gamma_{j}, 0\right)$ at time $t=0$ is given, then the number density $N\left(\gamma_{j}, \Delta t\right)$ can be calculated at time $t=\Delta t$. The iteration of above prescription gives the electron number density at an arbitrary time $t$ (e.g., Chaiberge \& Ghisellini 1999).

After calculating the electron number density $N(\gamma, t)$ at a time $t$, we can use the formulae given by Katarzynski et al. (2001) to calculate the synchrotron intensity $I_{\mathrm{s}}(v, t)$ and the intensity of self-Compton radiation $I_{\mathrm{c}}(v, t)$, and then calculate the flux density observed at the Earth as follows:

$$
F_{\text {tot }}(v, t)=\pi \frac{R^{2}}{d^{2}} \delta^{3}(1+z)\left[I_{\mathrm{s}}(v, t)+I_{\mathrm{c}}(v, t)\right],
$$

where $d$ is the luminosity distance, $z$ is the redshift, and $\delta=[\Gamma(1-\beta \cos \theta)]^{-1}$ is the Doppler factor where $\Gamma$ is the blob Lorentz factor, $\theta$ is the angle of the blob vector velocity to the line of sight and $\beta=v / c$. Since at high energies the Compton photons may produce pairs by interacting with the synchrotron photons, this process may be decrease the observed high energy radiation (Coppi \& Blandford 1990; Finke et al. 2009). Katarzynski et al. (2001) analyze the absorption effect due to pair-production inside the source, they found that its process is almost negligible. On the other hand, very high energy (VHE) photons from the source are attenuated by photons from the extragalactic background light (EBL). Therefore, after taking the absorption effect, the flux density 
observed at the Earth becomes

$$
F(v)=F_{\text {tot }}(v, t) \exp [-\tau(v, z)],
$$

where $\tau(v, z)$ is the absorption optical depth due to interactions with the EBL (Kneiske et al. 2004; Dwek \& Krennrich 2005). In our calculation, we use the absorption optical depth which is deduced by the average EBL model in Dwek \& Krennrich (2005).

\section{VALIDATION OF THE NUMERICAL CODE}

In order to validate our numerical code, we compare the time evolution of the electron spectrum calculated in our code with the analytic solutions given by Chang \& Cooper (1970). In our calculation, we assume that electrons lose energy by synchrotron and IC cooling, where the IC cooling is assumed to occur in the Thomson regime. Since the loss rates of both synchrotron radiation and inverse Compton scattering satisfy $d \gamma / d t \propto \gamma^{2}$, we can write the characteristic cooling time as $t_{\text {cool }}(\gamma)=1 / C_{0} \gamma$ with a cooling coefficient $C_{0}$. Otherwise, the system has not injection and escape of the particle during the evolution process (i.e. $Q(\gamma, t)=0$ and $E(\gamma, t)=0$ ). Under the above assumptions, equation (2) can be written as in the steady state $(\partial N(\gamma) / \partial t=0)$

$$
\frac{\partial}{\partial \gamma}\left[(C(\gamma)-A(\gamma)) N(\gamma)+D(\gamma) \frac{\partial N(\gamma)}{\partial \gamma}\right]=0,
$$

Chang \& Cooper (1970) gave the general solution of above equation as

$$
N(\gamma)=x \exp \left(-\int_{\gamma_{\min }}^{\gamma_{\max }} \frac{C(\gamma)-A(\gamma)}{D(\gamma)} d \gamma\right),
$$

where $x$ is a integration constant. Assuming $N_{\text {ini }}(\gamma)$ is the initial electron distribution between $\gamma_{0 \min }$ and $\gamma_{0 \max }$, total number of the particles in the system is given by $N_{\text {total }}=$ $\int_{\gamma_{0 \min }}^{\gamma_{0 \max }} N_{\text {ini }}(\gamma) d \gamma$, and then the integration constant can be determined by

$$
x=\frac{N_{\text {total }}}{\exp \left(-\int_{\gamma_{\min }}^{\gamma_{\max }} \frac{C(\gamma)-A(\gamma)}{D(\gamma)} d \gamma\right)},
$$

Using $C(\gamma)=C_{0} \gamma^{2}$ and $D(\gamma)=\gamma^{2} / 2 t_{\text {acc }}$, we can obtain

$$
N(\gamma)=x \gamma^{2} \exp \left(-2 C_{0} t_{\mathrm{acc}}(\gamma-1)\right) .
$$

At the equilibrium energy $\gamma_{\mathrm{e}}$, the acceleration process is fully compensated for by the cooling and then the acceleration time can be given by $t_{\mathrm{acc}}=1 / C_{0} \gamma_{\mathrm{e}}$. When the electrons energy $\gamma$ equals to $\gamma_{\mathrm{e}}$, Eq. (11) has a maximum value. In our tests, we adopt following values: $\gamma_{\mathrm{e}}=10^{4.5}, N_{\mathrm{ini}}=1 \mathrm{~cm}^{-3}$, and $C_{0}=3.48 \times 10^{-11} \mathrm{~s}^{-1}$.

In figure 1, we show the results of the particle number density $N(\gamma, t)$ at different evolution timescales calculated in our numerical code for three cases. For comparison, we also show the numerical values (black lines) in the steady state given by Eq. (18) for three cases. In the first case, we assume an initial electron distribution between $\gamma_{0 \min }=1$ and $\gamma_{0 \max }=2$, which means that the electron energy is much less than the equilibrium energy $\gamma_{\mathrm{e}}$ and electrons acceleration should dominate over all evolution processes. In this case, the electron number density of the system decreases in the initial energy range and simultaneously increases around the equilibrium as the evolution timescale increases. Until evolution timescale is

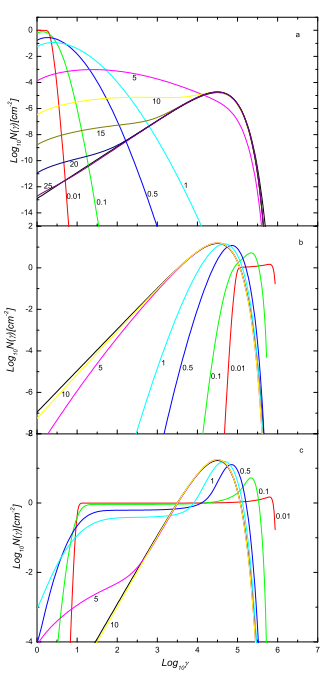

FIG. 1.- Numerical results of the particle number density $N(\gamma, t)$ at different evolution timescales calculated in our code for three cases. For comparison, the analytic solutions given by Eq. 18] are shown with black lines. The top panel(a) shows the initial electron distribution between $\gamma_{0 \min }=1$ and $\gamma_{0 \max }=2$ (case 1), middle panel(b) shows the initial electron distribution between $\gamma_{0 \min }=10^{5}$ and $\gamma_{0 \max }=10^{6}$ (case 2$)$, and bottom panel(c) shows the initial electron distribution between $\gamma_{0 \min }=10$ and $\gamma_{0 \max }=10^{6}$ (case 3). Marks near color lines represent the evolution timescales in units of $t_{\text {acc }}$.

increased to be more than $25 t_{\text {acc }}$, the system reaches the stationary Maxwellian distribution given by Eq. (18) (see top panel of Fig. 11). In the second case, we assume the initial electron distribution between $\gamma_{0 \min }=10^{5}$ and $\gamma_{0 \max }=10^{6}$, which indicates that the electron energy is about the equilibrium energy, electrons cooling should dominate over all evolution processes. When the evolution timescale increases to $10 t_{\text {acc }}$, the system reaches the stationary Maxwellian distribution given by Eq. (18) (see middle panel of Fig. 1). In the third case, we assume the initial electron distribution between $\gamma_{0 \min }=10$ and $\gamma_{0 \max }=10^{6}$. In this case, the evolution of the electron distribution with time depends on both electron cooling and acceleration processes and the system reaches the stationary Maxwellian distribution when the evolution timescale is about $10 t_{\text {acc }}$ (see bottom panel of Fig. 1). In a word, the electron spectra with larger evolution timescales $\left(t=20 t_{\text {acc }}\right.$ for the first case and $t \approx 10 t_{\text {acc }}$ for the second and third cases) calculated in our code are in agreement with the analytic solution (i.e. Eq. (18) given by Chang \& Cooper (1970).

\section{APPLY TO THE FLARING IN MARKAIN 501}

Using the time-dependent one-zone SSC solution for a spherical geometry, we can calculate $\mathrm{X}$-ray/TeV $\gamma$-ray spectra in the stable (pre-burst) and variable (in-burst) states. In order to do so, firstly we search for the steady state solution for electron and photon spectra. Assuming a constant initial electron distribution $N_{\text {ini }}(\gamma, 0)=2.1 \mathrm{~cm}^{-3}$ for $1 \leqslant \gamma \leqslant 2$, we calculate the time evolution of the spectra to $t=15 t_{\text {acc }}$, where the injection rate of the electron population is $Q(\gamma)=2.1 \mathrm{~cm}^{-3}$ $\mathrm{s}^{-1}$ for $1 \leqslant \gamma \leqslant 2$ and a constant escape for all evolution process are assumed. The parameters are used as follows: minimum and maximum Lorentz factors of electrons are $\gamma_{\min }=1$, $\gamma_{\max }=10^{7}$, magnetic field strength is $B=0.71 \mathrm{G}$, emission region size is $R=0.205 \times 10^{15} \mathrm{~cm}$, Doppler factor is $\delta=22.5$, 


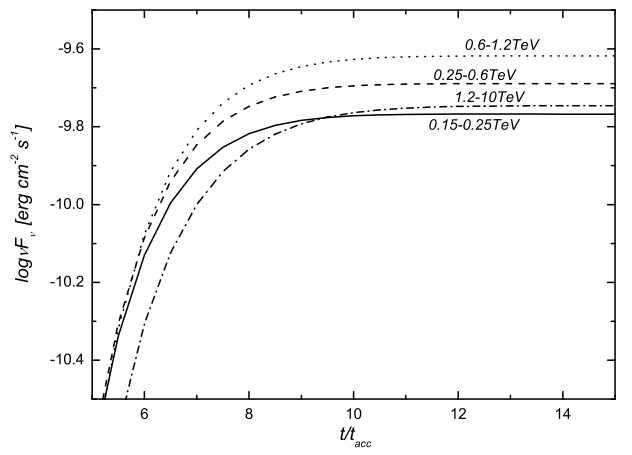

FIg. 2.- Predicted spectra at $0.15-0.25 \mathrm{TeV}, 0.25-0.6 \mathrm{TeV}, 0.6-1.2$ $\mathrm{TeV}$, and $1.2-10 \mathrm{TeV}$ in the time-dependent one-zone SSC model. When the evolution timescales $t \geqslant 10 t_{\text {acc }}$, steady state spectra are obtained.

and acceleration timescale $t_{\mathrm{acc}}=t_{\mathrm{esc}}=R / c$. In Fig. 2, we show the changes of calculating energy flux $v F_{v}$ at 4 energy bands of $0.15-0.25 \mathrm{TeV}, 0.25-0.6 \mathrm{TeV}, 0.6-1.2 \mathrm{TeV}$, and $1.2-10 \mathrm{TeV}$ with the evolution time normalized to the acceleration time. It can be seen from Fig. 2 that the steady states for all $\mathrm{TeV}$ energy bands can be reached when the evolution time $t \geq 10 t_{\text {acc }}$.

We assume that relativistic electrons are in the steady state during the pre-burst of X-rays and $\mathrm{TeV} \gamma$-rays. Therefore, we can calculate the pre-burst X-ray/ $\mathrm{TeV} \gamma$-ray spectrum in the one-zone SSC model using the steady state electron spectrum. In Fig 3, we show predicted pre-burst spectrum from X-ray to $\mathrm{TeV} \gamma$-ray bands (solid curve). For comparison, observed data of markarian 501 at X-ray band and TeV band on the July 9, 2005 (Albert et al. 2007) are also shown, where black solid circles with error bars represent the observed values at the preburst. It can be seen that the observed data in the pre-burst state can be reproduced in the SSC model.

We now consider the properties of $\mathrm{TeV} \gamma$-ray flare of markarian 501 in 2005 July. In order to do it, we use the physical parameters selected above and consider the resulting steady state spectrum as an initial condition, but we change the injection rate of the electron population to

$$
Q(\gamma)= \begin{cases}5.88 \mathrm{~cm}^{-3} \mathrm{~s}^{-1} & \text { for } 1 \leq \gamma \leq 2 \text { and } t \leq 0.5 t_{\mathrm{acc}}, \\ 2.1 \mathrm{~cm}^{-3} \mathrm{~s}^{-1} & \text { otherwise }\end{cases}
$$

Under above assumptions, we reproduce the observed $\mathrm{TeV}$ photon spectrum (dash curve) of markarian 501 on the July 9 , 2005 in Fig. 3. Furthermore we simulated the light curves at energy bands of $0.15-0.25 \mathrm{TeV}, 0.25-0.6 \mathrm{TeV}, 0.6-1.2 \mathrm{TeV}$, and $1.2-10 \mathrm{TeV}$ respectively and show the results in Fig. 4] where the fluxes are normalized to the pre-burst state. It can be seen that (1) the quasi-symmetric light curve during the flare is reproduced quite well; (2) the peaking time of the flare at higher energies lags relative to that at lower energies; and (3) the amplitude of the flare becomes smaller as the photon energy increase.

In order to comparing the simulated light curves with the observations, we show the comparisons of predicted light curves with the observations by MAGIC (Albert et al. 2008) in Fig. 5. The integrated fluxes in the left side of the vertical dashed lines of this figure are estimated using the differential spectra showed in Fig. 3. It can be seen from this figure that our model can reproduce the flare at the energy bands of 0.15

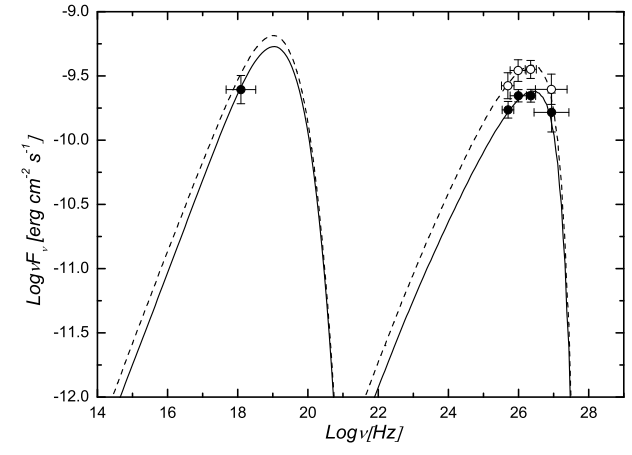

FIG. 3.- Comparisons of predicted multi-wavelength spectra with observed data of markarian 501 on the July 9, 2005. solid and dash curves represent steady state (or pre-burst) and the flaring (or in-burst) state spectra, respectively. Observed data come from Albert et al. (2007).

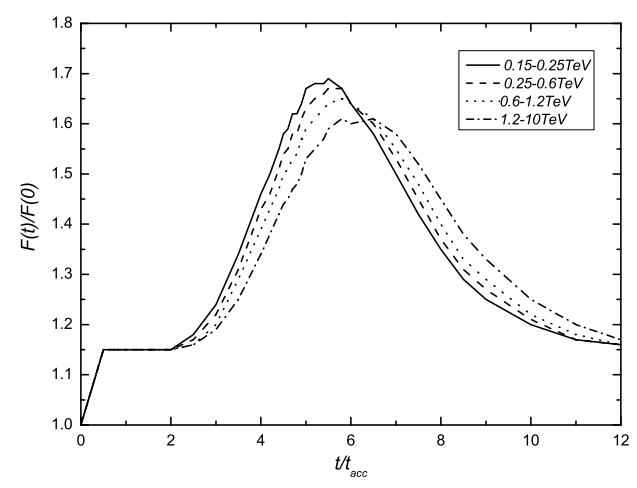

FIG. 4.- Simulated light curves at energy bands of $0.15-0.25 \mathrm{TeV}, 0.25$ - $0.6 \mathrm{TeV}, 0.6-1.2 \mathrm{TeV}$, and $1.2-10 \mathrm{TeV}$ for the rapid flare of markarian 501 on July 9, 2005. The fluxes at different wavelength are normalized to the pre-burst state value. The quasi-symmetric shape of the light curve and decreasing time lag of the peak with increasing energy are clearly seen.

- 0.25, $0.25-0.6,0.6-1.2$, and $1.2-10 \mathrm{TeV}$.

Finally, we calculate the time lag between $0.15-0.25 \mathrm{TeV}$ and $1.2-10 \mathrm{TeV}$ using the Gaussian fit of the simulated light curves, and find out that the light curve at higher energies (1.2 $-10 \mathrm{TeV})$ lags relative to that at lower energies $(0.15-0.25$ $\mathrm{TeV}$ ) by a factor of about $0.9 t_{\mathrm{acc}}$, this timescale correspond to about 4.7 minutes in the observer's frame. We also calculate the evolution of the hardness ratio which is defined as the ratio $\frac{F(1.2-10 \mathrm{TeV})}{F(0.6-1.2 \mathrm{TeV})}$. The evolution of the hardness ratio with the emitted flux above $1.2 \mathrm{TeV}$ is shown in Fig. 6. It can be seen from Fig. 6 that the evolution of the flare points shows a clear canonical anti-clockwise-loop.

\section{DISCUSSION AND CONCLUSIONS}

In this paper, we have tried to explain the $\mathrm{TeV} \gamma$-ray flare of markarian 501 observed by MAGIC telescope on July 9, 2005 in the context of the time-dependent one-zone SSC model which includes stochastic particle acceleration. In this model, particles with low energy are assumed to be injected and then are accelerated to higher energy by second-order Fermi acceleration mechanism (Fermi 1949), the most important photon targets for inverse Compton scattering by relativistic electrons are the synchrotron photons. We have studied time-dependent 


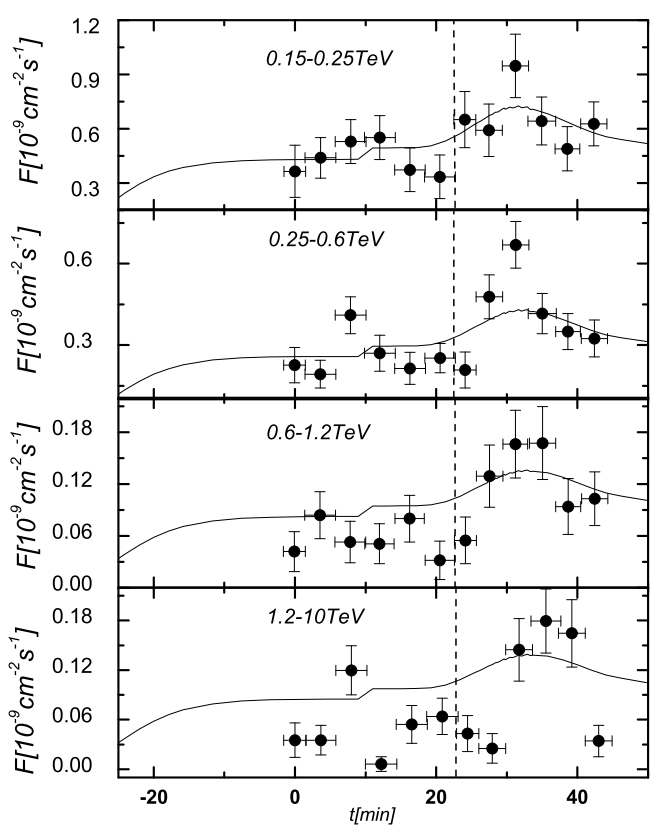

FIG. 5.- Comparisons of simulated light curves (solid lines) with observational light curve (data points) from the Albert et al. (2008) for the night July 9,2005 . The vertical dashed lines divide the light curves into steady (i.e., pre-burst) and variable (i.e., in-burst) states.

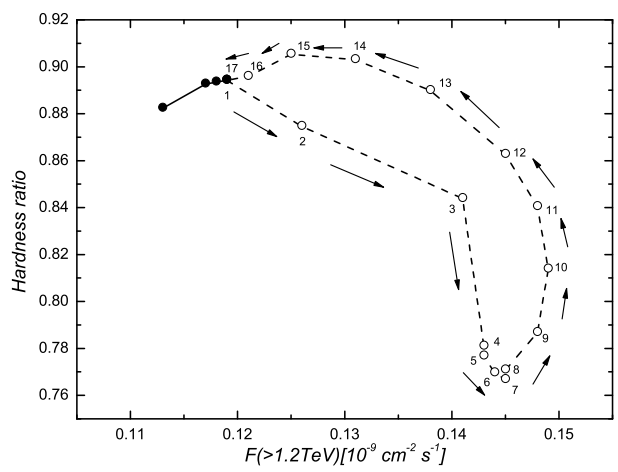

FIG. 6.- Simulated the hardness ratio $\frac{F(1.2-10 \mathrm{TeV})}{F(0.6-1.2 \mathrm{TeV})}$ versus flux $F(>1.2 \mathrm{TeV})$ for the night July 9, 2005. Black and open circles represent pre-burst and inburst emission respectively. The number near the markers denote the position of the points in the light curves. A canonical anti-clockwise-loop is seen.

properties of flares by reproducing the pre-burst spectrum of the source and varying the injection rate. In our results, the behaviour of the mean multi-frequencies spectra before and during the flare is a little different, the peaks of both synchrotron and IC emissions move to lower frequencies, we argue that this can be explained by the energy loss of the electrons during the outburst. In this scenario, the hard lag flare can be obtained and during the flare, it shows a clear canonical anticlockwise-loop.

It should be noted that hard lags require some sort of particle acceleration. If the variability timescale is faster than the cooling timescale, the radiation from accelerated particles would show a hard lag (Albert et al. 2008). Kirk et al. (1998) argued that the hard lag from the acceleration process induces to the anti-clockwise-loop pattern. In this view, Albert et al. (2007) concluded that the acceleration process of low energy particles probably dominate over the $\mathrm{TeV} \gamma$-ray flare. Assuming a low energy electrons injection and stochastic acceleration, our calculations predicted hard lags dependent flaring activity and showed a anti-clock-loop evolution of the hardness ratio with the flux. These are in agreement with the observational results on July 9, 2005, and imply that, during the flare, the dynamics of the system is dominated by the acceleration, rather than by the cooling processes. However, a detailed investigation of electron acceleration in the presence of losses has so far been performed only by a few investigators (e.g. Mastichiadis \& Moraits 2008). Given the complexity of the flaring activity of high energy radiation, this requires more detailed observations and the issue to be open.

The magnetohydrodynamic turbulence will is generated if standing shocks form in the neighborhood of the central object, which amplify any incoming upstream turbulence in the downstream accretion shock magnetosheath (Campeanu $\&$ Schlickeiser 1992). These magnetohydrodynamic plasma waves are the free energy and lead to stochastic acceleration of charged particles. Actually, stochastic acceleration occurs wherever there are turbulent magnetic fields and can spread to an extended region, the size is determined by the turbulence generation and decay rates. Virtanen \& Vainio (2005) simulated the stochastic acceleration in relativistic shocks and shows, when the particles were accelerated behind the discontinuity, a gradual shift of the whole particle spectrum to higher energy. Some recent observations of particles spectra with hard power law spectral indices, $N(\gamma) \propto \gamma^{-n}$ with $n<2$, suggests that the stochastic acceleration is seen in the observations (Katarzynski et al. 2006; Böttcher et al. 2008). The model presented here contains the stochastic acceleration process. For simplicity, we introduced a constant acceleration term, which is associated with the momentum diffusion coefficient $D(p, t)$. The form of the diffusion coefficient due to interactions with magnetohydrodynamic waves has been discussed in details (e.g. Kulsrud \& Ferrari 1971; Schlickeiser 2002). In our model, both constant acceleration and escape times are assumed, leading to $D(\gamma, t)=\gamma^{2} / 2 t_{\text {acc }} \propto \gamma^{2}$. The form of the diffusion coefficient corresponds to the hard-sphere approximation, in which the mean free path for article-wave interaction is independent of particle energy, and probably induces to a complicated spectrum. Furthermore, since the basic shock acceleration models postulate that $t_{\text {acc }} \simeq t_{\text {esc }}$ (e.g., Katarzynski et al. 2006), we adopt the shorter acceleration and escape timescales $\left(t_{\mathrm{acc}}=t_{\mathrm{esc}}=\frac{R}{c}=t_{\mathrm{cr}}\right)$ than other investigators (generally, $t_{\mathrm{acc}}>t_{\mathrm{cr}}$, and $t_{\mathrm{esc}}>t_{\mathrm{cr}}$, see e.g., Kirk et al. 1998; Mastichiadis \& Moraitis 2008). These assumptions can lead to higher acceleration rate and lower escape rate, and make more particles acceleration up to high energy rapidly.

There are two scenarios for explaining the intrinsic variability. The first scenario assumes that the observed variations origin from the geometry of emitting sources (e.g., Camenzind \& Krockenberger 1992; Gopal-Krishna \& Wiita 1992). The second scenario assumes that the variability is generated by change of the emission condition. A typical example is that fresh particles are injected into acceleration region and then are accelerated (e.g., Blandford \& Konigl 1979; Marscher \& Gear 1985; Celotti et al. 1991; Kirk et al. 1998). In order to reproduce both high energy radiation and variability of markarian 501, we change the injection rate of the low energy particles. It should be noted that when the shock front over- 
runs a region in the jet in which the local plasma density is enhanced. The number of particles increase as an avalanche occurring in the jet, the injection rate can be expected to change.

\section{ACKNOWLEDGMENTS}

We thank the anonymous referee for valuable comments and suggestions. This work is partially supported by the
National Natural Science Foundation of China under grants 10763002 and 10778702 and the Natural Science Foundation of Yunnan Province under grants 2009ZC056M, 2008CC011. This work is also supported by the Science Foundation of Yunnan educational department (grant 08Z0020).

\section{REFERENCES}

Aharonian, F., Akhperjanian, A. G., Bazer-Bachi, A. R., et al. 2007, ApJ, 664, L71

Albert, J., Aliu, E., Anderhub, H., et al. 2007, ApJ, 669, 862

Albert, J., Aliu, E., Anderhub, H., et al. 2008, Phys. Lett. B, 668, 253

Bednarek, W., \& Wagner, R. M., 2008, A\&A, 486, 679

Begelman, M. C., Fabian, A. C., \& Rees, M. J., 2008, MNRAS, 384, L19

Blandford, R. D., \& Konigl, A., 1979, ApJ, 232, 34

Böttcher, M., Dermer, C. D., \& Finke, J. D., 2008, ApJ, 679, L9

Camenzind, M., \& Krockenberger, M., 1992, A\&A, 255, 59

Campeanu, A., \& Schlickeiser, R., 1992, A\&A, 263, 413

Celotti, A., Maraschi, L., \& Treves, A., 1991, ApJ, 377, 403

Chang, J. S., \& Copper, G., 1970, Computational Physics, 6, 1

Chaiberge, M., \& Ghisellini, G., 1999, MNRAS, 306, 551

Coppi, P. S., \& Blandford, R. D., 1990, MNRAS, 245, 453

Dwek, E., \& Krennrich, F., 2005, ApJ, 618, 657

Fermi, E., 1949, Phys. Rev., 75, 1169

Finke J. D., Dermer, C. D., \& Bottcher, M., 2009, ApJ, 686, 181

Gopal-Krishna, \& Wiita, P. J., 1992, A\&A, 259, 109

Kataoka, J., Takahashi, T., Wagner, S. J., et al. 2001, ApJ, 560, 659

Katarzynski, K., Sol, H., \& Kus, A., 2001, A\&A, 367, 809

Katarzynski, K., Ghisellini, G., Mastichiadis, A., et al. 2006, A\&A, 453, 47
Kirk, J. G., Rieger, F. M., \& Mastichiadis, A., 1998, A\&A, 333, 452 Kirk, J. G., \& Mastichiadis, A., 1999, Astroparticles Physics, 11, 45

Kneiske, T. M., Bretz, T., Mannheim, K., et al. 2004, A\&A, 413, 807

Kulsrud, R. M., \& Ferrari, A., 1971, Ap\&SS, 12, 302

Kusunose, M., Takahara, F., \& Li, H., 2000, ApJ, 536, 299

Lainela, M., et al. 1999, ApJ, 521, 561

Marscher, A. P., \& Gear, W. K., 1985, ApJ, 298, 114

Mastichiadis, A., \& Moraitis, K., 2008, A\&A, 491, L37

Moderski, R., Sikora, M., Coppi, P. S., et al. 2005, MNRAS, 363, 954

Nakar, E., Ando, S., \& Sari, R., 2009, ApJ, 703, 675

Park, B. T., \& Petrosian, V., 1995, ApJ, 446, 699

Park, B. T., \& Petrosian, V., 1996, ApJS, 109, 255

Press, W. H., Numerical Recipes in Fortran, Cambridge Univ. Press, Cambridge

Schlickeiser R., 2002, Cosmic Ray Astrophysics, Berlin: Springer

Sillanpää, A., Mikkola, S., \& Valtaoja, L., 1991, A\&AS, 88, 225

Tammi, J., \& Duffy, P., 2009, MNRAS, 393, 1063

Tverskoi, B. A., 1967, Sovient Phys. JETP, 25, 317

Virtanen, J. J. P., \& Vainio, R., 2005, ApJ, 621, 313

Wagner, S. J., \& Witzel, A., 1996, AJ, 111, 2187 